TRANSACTIONS OF THE

AMERICAN MATHEMATICAL SOCIETY

Volume 349, Number 3, March 1997, Pages 935-954

S 0002-9947(97)01806-0

\title{
THE HOMOLOGY REPRESENTATIONS OF THE $k$-EQUAL PARTITION LATTICE
}

\author{
SHEILA SUNDARAM AND MICHELLE WACHS
}

\begin{abstract}
We determine the character of the action of the symmetric group on the homology of the induced subposet of the lattice of partitions of the set $\{1,2, \ldots, n\}$ obtained by restricting block sizes to the set $\{1, k, k+1, \ldots\}$. A plethystic formula for the generating function of the Frobenius characteristic of the representation is given. We combine techniques from the theory of nonpure shellability, recently developed by Björner and Wachs, with symmetric function techniques, developed by Sundaram, for determining representations on the homology of subposets of the partition lattice.
\end{abstract}

\section{INTRODUCTION}

For $2 \leq k \leq n$, the $k$-equal partition lattice $\Pi_{\left(k, 1^{n-k}\right)}$ is defined to be the join-sublattice of the partition lattice $\Pi_{n}$ generated by set partitions consisting of $n-k$ blocks of size one and one block of size $k$. In other words, $\Pi_{\left(k, 1^{n-k}\right)}$ is the joinsublattice of $\Pi_{n}$ consisting of partitions having no blocks of sizes $2,3, \ldots, k-1$. This lattice was first studied by Björner, Lovász and Yao in [Bj-Lo-Y] in connection with a problem in complexity theory. It was then investigated by Björner and Welker, who showed in $[\mathrm{Bj}-\mathrm{We}]$ that any lower interval in $\Pi_{\left(k, 1^{n-k}\right)}$ has the homotopy type of a wedge of spheres of varying dimensions, and determined the Betti numbers.

The present paper is devoted to a study of the representation of the symmetric group $S_{n}$ on the homology of $\Pi_{\left(k, 1^{n-k}\right)}$. For $k=2$, the action of the symmetric group on the homology of $\Pi_{\left(k, 1^{n-k}\right)}=\Pi_{n}$, has been extensively studied in recent years beginning with work of Stanley [St] and Hanlon [Ha1]. One motivation for interest in the homology representation of $\Pi_{n}$ stems from its relationship with the free Lie algebra (cf. [Bar, Jo, Wa2]). Another comes from its fundamental role in the theory of hyperplane arrangements as developed by Orlik and Solomon [O-So] (see [Le-So]). The lattice $\Pi_{n}$ is the intersection lattice of the braid arrangement studied by $\mathrm{Arnol}^{\prime} \mathrm{d}[\mathrm{Ar}]$ and Brieskorn $[\mathrm{Br}]$. For general $k$, the lattice $\Pi_{\left(k, 1^{n-k}\right)}$ arose as the intersection lattice for a natural generalization of the braid arrangement called the $k$-equal subspace arrangement (see $[\mathrm{Bj}-\mathrm{Lo}-\mathrm{Y}]$ and $[\mathrm{Bj}-\mathrm{We}])$.

The lattice $\Pi_{\left(k, 1^{n-k}\right)}$ is not ranked. Much of the intricacy in studying the topology of this lattice is due to the fact that the standard combinatorial techniques were developed with ranked posets in mind. We shall apply the powerful theory of shellability, recently extended to nonranked posets by Björner and Wachs [Bj-Wa],

Received by the editors April 20, 1994.

1991 Mathematics Subject Classification. Primary 05E25, 06A08, 06A09; Secondary 05E05, 05E10, 20C30, 05A18, 52B30.

Supported in part by NSF grants DMS 9102760 and DMS 9311805. 
to the lattice $\Pi_{\left(k, 1^{n-k}\right)}$. The shelling of the $k$-equal lattice presented in Section 1 gives a vector space decomposition of the homology of the lattice into a direct sum of homology of more familiar posets. This leads to a decomposition for the $S_{n}$-module structure of the homology, which is presented in Theorem 2.8.

The theory of symmetric functions ([Macd]) allows us to carry out representationtheoretic calculations in a compact manner. Using the techniques developed in [Su1] for determining representations of $S_{n}$ on the homology of subposets of the partition lattice, in Section 3 we determine the $S_{n}$-action on each graded piece of the homology of $\Pi_{\left(k, 1^{n-k}\right)}$ (Theorem 3.5). The Frobenius characteristics of these modules have an elegant expression as a plethysm of the representation on the homology of the usual partition lattice, with irreducibles indexed by hooks. We also compute the Frobenius characteristic of the $S_{n}$-action on the Whitney homology ([Bac, Bj2]), that is, the homology of lower intervals of $\Pi_{\left(k, 1^{n-k}\right)}$. The result of this computation (Theorem 3.7) is more intricate than the expression for the homology of $\Pi_{\left(k, 1^{n-k}\right)}$; however, as shown in $[\mathrm{SW}]$, it is an essential ingredient in determining the representation of $S_{n}$ on the cohomology of the complement of the $k$-equal arrangement. In particular in $[\mathrm{SW}]$ this computation is applied to the study of the related orbit space.

The characters which arise in this paper are closely related to the character of $S_{n}$ on the multilinear part of the free Lie algebra. For $k=2$ the basis for cohomology provided by the shelling gives an $S_{n}$-equivariant filtration of the top cohomology of the partition lattice, which corresponds to Reutenauer's filtration of the free Lie algebra by derived series [Re]. For $k \geq 2$, our decomposition of the representation on the cohomology of the $k$-equal lattice is thus a generalisation of the derived series decomposition. These and other representation-theoretic aspects are discussed in Section 4 . In particular the case $k=2$ gives rise to a curious decomposition of the regular representation of $S_{n}$.

The authors would like to thank Anders Björner and Volkmar Welker for stimulating discussions.

\section{A Shelling For the LATtice $\Pi_{\left(k, 1^{n-k}\right)}$.}

We begin by reviewing some basic notions in the theory of shellable posets. For ranked posets, Björner [Bj1] introduced the powerful notion of lexicographic shelling, which was generalised by Björner and Wachs [Bj-Wa] to arbitrary posets. We now define the notion of a lexicographic shelling. A labelling $\lambda$ of a poset is a mapping that assigns, to the edges of the Hasse diagram (i.e. of the covering relations) of the poset, elements of a linearly ordered set. The linearly ordered set is called the label set of the labelling.

For each unrefinable chain $x_{0}<x_{1}<\cdots<x_{n}$ there is a corresponding label sequence $\lambda\left(x_{0}, x_{1}\right), \ldots, \lambda\left(x_{n-1}, x_{n}\right)$. The lexicographic ordering on sequences of elements of the label set induces an ordering on the unrefinable chains from $x$ to $y$ in any interval $[x, y]$ of the poset. We say that an unrefinable chain $x_{0}<x_{1}<\cdots<x_{n}$ is an increasing (resp. decreasing) chain from $x_{0}$ to $x_{n}$ (with respect to the labelling $\lambda$ ) if the sequence of labels $\lambda\left(x_{0}, x_{1}\right), \ldots, \lambda\left(x_{n-1}, x_{n}\right)$ is a strictly increasing (weakly decreasing) sequence in the label set.

(1.1). ( [Bj-Wa]) Define a lexicographic shelling of an arbitrary poset, with unique maximal and minimal elements, $\hat{1}$ and $\hat{0}$ respectively, to be a labelling which satisfies the following key conditions: 
(E1) every interval $[x, y]$ has a unique increasing unrefinable chain from $x$ to $y$, and

(E2) this chain is least, with respect to the total order induced on the chains as described above, among all unrefinable chains from $x$ to $y$.

The original version of this condition, for ranked posets, is due to [Bj1, Definition 2.2] and requires the existence in each interval of a unique weakly increasing chain instead of a strictly increasing chain. For ranked posets, the definition used above was first introduced in ([Wa1, Theorem 6.2]).

The order complex of a poset $P$ with $\hat{0}$ and $\hat{1}$, denoted $\Delta(P)$, is the simplicial complex whose faces are the chains of $P-\{\hat{0}, \hat{1}\}$. The following theorem summarises the crucial topological information provided by a lexicographic shelling of a poset.

Proposition (1.2) [Bj-Wa]. Let $P$ be any poset equipped with a lexicographic shelling as defined above.

(i) Then $\Delta(P)$ has the homotopy type of a wedge of spheres. For each $i \geq-1$, the number of spheres of dimension $i$ is the number of decreasing chains from $\hat{0}$ to $\hat{1}$ in the poset, of length $i+2$.

(ii) The decreasing chains form a basis for the cohomology of P. More precisely, the collection of simplices $\left(x_{1}<\cdots<x_{n}\right)$, where $\hat{0}<x_{1}<\cdots<x_{n}<\hat{1}$ is a decreasing chain from $\hat{0}$ to $\hat{1}$ in $P$, form a basis for the cohomology of the order complex $\Delta(P)$.

For any poset $P$ with $\hat{0}$ and $\hat{1}$, let $\widetilde{H}_{i}(P)$ (resp. $\left.\widetilde{H}^{i}(P)\right)$ denote the reduced simplicial homology (resp. cohomology) of $\Delta(P)$ in dimension $i$, over $\mathbf{C}$. Also let $\widetilde{H}_{*}(P)$ be the direct sum $\bigoplus_{i} \widetilde{H}_{i}(P)$ and $\widetilde{H}^{*}(P)$ be the $\operatorname{direct} \operatorname{sum} \bigoplus_{i} \widetilde{H}^{i}(P)$. For intervals $[x, y]$ in $P$, let $\Delta(x, y), \widetilde{H}_{-}(x, y)$ and $\widetilde{H}^{-}(x, y)$ denote the order complex, homology and cohomology of $[x, y]$, respectively.

Since the (co)homology of $P$ is taken over a field, there is a natural vector space isomorphism (via duality) between homology and cohomology. Moreover, if $G$ is a group of automorphisms of the poset $P$ then $G$ acts on $\widetilde{H}_{i}(P)$ and on $\widetilde{H}^{i}(P)$ and these representations of $G$ are dual. Since dual representations of the symmetric group are isomorphic, the homology and cohomology of $\Pi_{\left(k, 1^{n-k}\right)}$ are therefore isomorphic $S_{n}$-modules. The use of cohomology will render our arguments more transparent, thanks to a simple description of the basis for cohomology provided by the shelling of $\Pi_{\left(k, 1^{n-k}\right)}$ described below.

Now we describe the shelling for the poset $\Pi_{\left(k, 1^{n-k}\right)}$. Consider the totally ordered set of labels

$$
\overline{1}<\overline{2}<\cdots<\bar{n}<1<2<\cdots<n .
$$

Suppose $x$ is covered by $y$ in $\Pi_{\left(k, 1^{n-k}\right)}$. Assign a label $\lambda(x, y)$ to the edge $x \rightarrow y$, according to one of the three possible covering relations, as follows: If $y$ is obtained from $x$ by

(i) creating a new $k$-block $B$ (i.e., a block $B$ of size $k$ ), then $\lambda(x, y)=\max (B)$;

(ii) merging a non-singleton block $B$ of $x$ with a singleton block $\{a\}$, then $\lambda(x, y)=$ $a$;

(iii) merging two non-singleton blocks $B_{1}, B_{2}$, then $\lambda(x, y)=\overline{\max \left(B_{1} \cup B_{2}\right)}$.

Proposition (1.3) [Bj-Wa]. The labelling described above is a lexicographic shelling of $\Pi_{\left(k, 1^{n-k}\right)}$. 


\section{The homology OF $\Pi_{\left(k, 1^{n-k}\right)}$.}

Given a lexicographic shelling of a poset, the combinatorial techniques of Proposition 1.2 provide bases for the cohomology of the corresponding order complexes. The crucial problem now is to identify the weakly decreasing chains in the poset. In this section we identify the decreasing chains in the labelling given for $\Pi_{\left(k, 1^{n-k}\right)}$ in Section 1. The following lemma provides the key information. For any maximal chain $\hat{0}=w_{0}<w_{1}<\cdots<w_{s}=\hat{1}$ in $\Pi_{\left(k, 1^{n-k}\right)}$, define the pivot of the chain to be the minimal element $w_{i}$ of the chain all of whose blocks sizes are $\geq k$. For any $w$ in $\Pi_{\left(k, 1^{n-k}\right)}$, let $\Delta(\hat{0}, w)_{\mathcal{B}}$ be the subcomplex of $\Delta(\hat{0}, w)$ generated by all maximal chains of $[\hat{0}, w]$ which have only non-barred labels. It follows easily that $\Delta(\hat{0}, w)_{\mathcal{B}}$ is the order complex of a partially ordered set $[\hat{0}, w]_{\mathcal{B}}$ which is in fact a subposet of $[\hat{0}, w]$.

Lemma (2.1). The decreasing chains from $\hat{0}$ to $\hat{1}$ in $\Pi_{\left(k, 1^{n-k}\right)}$ are in one-to-one correspondence with pairs of unrefinable chains $\left(c_{1}, c_{2}\right)$ such that $c_{1}$ is decreasing from $\hat{0}$ to $w$ in $[\hat{0}, w]_{\mathcal{B}}, c_{2}$ is decreasing from $w$ to $\hat{1}$ in $[w, \hat{1}]$ and $w$ is a partition in $\Pi_{\left(k, 1^{n-k}\right)}$ with no singleton blocks. The correspondence is

$$
c \mapsto\left(c_{1}, c_{2}\right),
$$

where $c_{1}$ is the portion of the chain $c$ in $\left[\hat{0}, w_{c}\right]$ and $c_{2}$ is the portion of $c$ in $\left[w_{c}, \hat{1}\right]$, $w_{c}$ being the pivot of the chain $c$.

In particular, the length of a maximal decreasing chain in $\Pi_{\left(k, 1^{n-k}\right)}$ is $(n-1-$ $t(k-2)$ ), where $t$ is the number of blocks in the pivot of the chain (so $\left.1 \leq t \leq\left\lfloor\frac{n}{k}\right\rfloor\right)$, and hence contributes to cohomology in dimension $(n-3-t(k-2))$.

Proof. Let $c=\left(\hat{0}=x_{0}<x_{1}<\cdots<x_{s}=\hat{1}\right)$ be a decreasing chain in $\Pi_{\left(k, 1^{n-k}\right)}$. We claim that if $x_{i}$ is the pivot $w_{c}$ of the chain $c$ then all labels on edges $x_{j-1}<x_{j}$ for $j \leq i$ are non-barred and all labels on edges $x_{j}<x_{j+1}$ for $j \geq i$ are barred. Indeed, the label sequence of the subchain $x_{i}<\cdots<x_{s}=\hat{1}$ consists entirely of barred labels, since at each step the only possible operation is to merge two nonsingleton blocks. Since $x_{i}$ is the pivot of the chain, the element $x_{i-1}$ contains at least one singleton block. Therefore $x_{i}$ is constructed from $x_{i-1}$ by either creating a $k$-block or merging a singleton with a non-singleton block. In either case the label of $x_{i-1}<x_{i}$ is non-barred. Since the chain $\hat{0}=x_{0}<x_{1}<\cdots<x_{s}=\hat{1}$ is decreasing, it follows that all labels on edges $x_{j-1}<x_{j}, j \leq i$, are also non-barred.

Since all the labels of $c$ that are below the pivot are non-barred, $c_{1}$ is in $\left[\hat{0}, w_{c}\right]_{\mathcal{B}}$. Also the subchains $c_{1}$ and $c_{2}$ are clearly decreasing. Hence $c$ splits into decreasing chains in $\left[\hat{0}, w_{c}\right]_{\mathcal{B}}$ and $\left[w_{c}, \hat{1}\right]$ as asserted.

Conversely, if $w$ is a partition with no singleton blocks, $c_{1}$ is decreasing from $\hat{0}$ to $w$ in $[\hat{0}, w]_{\mathcal{B}}$ and $c_{2}$ is decreasing from $w$ to $\hat{1}$ in $[w, \hat{1}]$ then the concatenation of the two chains must be decreasing since all the labels on $c_{1}$ are non-barred and all the labels on $c_{2}$ are barred. Since the pivot of the concatenation is clearly $w$, the correspondence given is indeed a bijection.

This lemma allows us to filter the decreasing chains according to the number of non-singleton blocks of its pivot elements. It transpires that this filtration is closely related to Hanlon's filtration ( [Ha2, equation (4.4.4)]) of the order complex of a poset with respect to a ranked order ideal. 
The first part of the following lemma is immediate; the second part follows from Proposition 1.2(ii) and the fact that a lexicographic shelling of a poset restricts to a lexicographic shelling of any interval.

Lemma (2.2). Suppose $w$ is a partition in $\Pi_{\left(k, 1^{n-k}\right)}$ with $t$ blocks all of size at least $k$. Then

(i) the interval $[w, \hat{1}]$ in $\Pi_{\left(k, 1^{n-k}\right)}$ is lattice-isomorphic to $\Pi_{t}$, and is therefore homotopy equivalent to a wedge of $(t-1)$ ! spheres of dimension $(t-3)$.

(ii) the decreasing chains from $w$ to $\hat{1}$ form a basis for $\widetilde{H}^{t-3}(w, \hat{1})$ and are determined at each step by merging the block containing the largest element $n$ with any other non-singleton block. (Note that the label sequence here consists entirely of $\bar{n}$ 's.)

We remark that the decreasing chains from $w$ to $\hat{1}$ described in Lemma 2.2 are the decreasing chains corresponding to the splitting basis and lexicographic shelling $\lambda_{2}$ of $\Pi_{t}$ given in [Wa1].

We introduce some definitions and notation.

Write $\mathcal{B}_{r}$ for the Boolean lattice of subsets of a set of $r$ elements, and $\mathcal{B}_{r}^{\geq k}$ for the truncated subposet of $\mathcal{B}_{r}$ consisting of the subsets of size at least $k$, together with an artificially attached least element. Note that $\mathcal{B}_{r}^{\geq k}$ is a subposet of rank $r-k+1$, obtained from the Boolean lattice by rank selection.

Let $\mu=\left(b_{1} \leq b_{2} \leq \cdots \leq b_{t}\right)$ be a partition of $n$. Let $N_{S_{n}}\left(S_{\mu}\right)$ denote the normaliser, in $S_{n}$, of the Young subgroup

$$
S_{\mu}=S_{\left\{1, \ldots, b_{1}\right\}} \times S_{\left\{b_{1}+1, \ldots, b_{2}\right\}} \times \cdots \times S_{\left\{b_{1}+\cdots+b_{t-1}+1, \ldots, n\right\}},
$$

where $S_{A}$ denotes the symmetric group on the elements of the set $A$. This is isomorphic to the direct product of wreath products $\chi_{j} S_{m_{j}}\left[S_{j}\right]$, where $m_{j}$ is the multiplicity of the part $j$ in $\mu$ and $S_{m}[G]$ denotes the wreath product of the symmetric group $S_{m}$ with the group $G$.

Let $P_{t}$ denote the set of integer partitions of $n$ with exactly $t$ parts all of size $\geq k$. We say that a partition $w \in \Pi_{n}$ has type $\mu$ if its multiset of block sizes form the integer partition $\mu$. For $\mu \in P_{t}$, let $w_{\mu}$ be the partition of type $\mu$ that is stabilised by $N_{S_{n}}\left(S_{\mu}\right)$. Let $[\hat{0}, \mu]_{\mathcal{B}}$ and $[\mu, \hat{1}]$ denote the subposets $\left[\hat{0}, w_{\mu}\right]_{\mathcal{B}}$ and $\left[w_{\mu}, \hat{1}\right]$, respectively. Clearly these subposets are invariant under the action of $N_{S_{n}}\left(S_{\mu}\right)$. We also let $\widetilde{H}^{*}(\hat{0}, \mu)_{\mathcal{B}}$ and $\widetilde{H}^{*}(\mu, \hat{1})$ denote $\widetilde{H}^{*}\left(\hat{0}, w_{\mu}\right)_{\mathcal{B}}$ and $\widetilde{H}^{*}\left(w_{\mu}, \hat{1}\right)$, respectively. These spaces are also $N_{S_{n}}\left(S_{\mu}\right)$-invariant.

Lemma (2.3). (i) Let $\mu=\left(b_{1} \leq b_{2} \leq \cdots \leq b_{t}\right) \in P_{t}$. The poset $[\hat{0}, \mu]_{\mathcal{B}}$ is $N_{S_{n}}\left(S_{\mu}\right)$ isomorphic to the direct product of posets $\bar{X}_{i=1}^{t} B_{b_{i}}^{\geq k}$. Consequently the subcomplex $\Delta(\hat{0}, \mu)_{\mathcal{B}}$ is $N_{S_{n}}\left(S_{\mu}\right)$-homotopy equivalent to the order complex of $\chi_{i=1}^{t} B_{b_{i}}^{\geq k}$.

(ii) Let $w \in \Pi_{\left(k, 1^{n-k}\right)}$ have no singleton blocks. Then the lexicographic shelling of $[\hat{0}, w]$ as an interval of $\Pi_{\left(k, 1^{n-k}\right)}$ restricts to a lexicographic shelling of the subposet $[\hat{0}, w]_{\mathcal{B}}$. Consequently the decreasing chains of $[\hat{0}, w]$ belonging to $[\hat{0}, w]_{\mathcal{B}}$ form a basis for $\widetilde{H}^{*}(\hat{0}, w)_{\mathcal{B}}$.

Proof. (i) The poset isomorphism follows from the fact that in $[\hat{0}, \mu]_{\mathcal{B}}$ all elements arise from $w_{\mu}$ by either splitting a $k$-block into its singletons or breaking off a singleton from a block of size $\geq k+1$. Clearly all partitions which are constructed in this way lie in $[\hat{0}, \mu]_{\mathcal{B}}$. The fact that this commutes with the action of $N_{S_{n}}\left(S_{\mu}\right)$ is clear. 
(ii) Let $x<y$ be elements of $[\hat{0}, w]_{\mathcal{B}}$. It is easy to see that the unique increasing chain from $x$ to $y$ in $[\hat{0}, w]$ with respect to the lexicographic shelling is in fact a chain in $[\hat{0}, w]_{\mathcal{B}}$. Hence the shelling on $[\hat{0}, w]$ restricts to a shelling on $[\hat{0}, w]_{\mathcal{B}}$, (see $[\mathrm{Bj} 1$, Proposition 2.8]) and the chains contributing to cohomology in $[\hat{0}, w]_{\mathcal{B}}$ are precisely the decreasing chains in $[\hat{0}, w]$ with no barred labels.

The shelling of $\Pi_{\left(k, 1^{n-k}\right)}$ allows one to recover the results of Björner and Welker [Bj-We, Theorem 4.5] on the homotopy type and Betti numbers of $\Pi_{\left(k, 1^{n-k}\right)}$ (see [Bj-Wa]). Furthermore, Lemmas 2.1, 2.2 and 2.3 give the following interpretation of the Betti numbers:

Proposition (2.4). As a vector space the cohomology of $\Pi_{\left(k, 1^{n-k}\right)}$ is isomorphic to the direct sum

$$
\bigoplus_{t} \bigoplus_{\mu=\left(b_{1} \leq \cdots \leq b_{t}\right) \in P_{t}} \bigoplus_{w: t y p e(w)=\mu}\left(\widetilde{H}^{*}\left(\searrow_{i=1}^{t} \mathcal{B}_{b_{i}}^{\geq k}\right) \otimes \widetilde{H}^{*}\left(\Pi_{t}\right)\right) .
$$

The vector space decomposition of Proposition 2.4 suggests that as an $S_{n^{-}}$ module, the cohomology of $\Pi_{\left(k, 1^{n-k}\right)}$ decomposes into a direct sum, indexed by $t$ and $\mu \in P_{t}$, of $S_{n}$-invariant modules, each of which is induced from a tensor product of the cohomology of a product of truncated Boolean lattices with the cohomology of a partition lattice. Our aim now is to prove that such a decomposition does indeed hold.

Although the next two technical lemmas pertain to all $k \geq 2$, they are needed only in order to deal with the case $k=2$.

Lemma (2.5). Let $w \in \Pi_{\left(k, 1^{n-k}\right)}$ be a partition all of whose blocks have size at least $k$, and let $(x<y<z)$ be an unrefinable chain in $[\hat{0}, w]_{\mathcal{B}}$. Let $c$ denote the chain $(x<z)$. If $\delta$ denotes the coboundary operator of the interval $[x, z]$ in $\Pi_{\left(k, 1^{n-k}\right)}$, then $\delta(c)$ is a linear combination of chains in $\Pi_{\left(k, 1^{n-k}\right)}$, which lie in $[\hat{0}, w]_{\mathcal{B}}$ in all cases except when $k=2$ and $z$ differs from $x$ in exactly one block $B_{z}$, and $B_{z}$ is the union of a block $B_{x}$ of $x$ with two singletons of $x$.

Proof. The hypothesis implies that the chain $x<y<z$ has no barred labels. Since $z$ has at most two non-singleton blocks which are different from those of $x$, there are five cases to consider:

(i) There are two blocks of size $k$ in $z$ all of whose elements are singletons in $x$.

(ii) There is a block $B_{z}$ of size $k+1$ in $z$ all of whose elements are singletons in $x$.

(iii) There is a block $B_{z}$ of size $k$ in $z$ all of whose elements are singletons in $x$, and a block $B_{z}^{\prime}$ in $z$ such that exactly one element of $B_{z}^{\prime}$ is a singleton in $x$.

(iv) There are two blocks $B_{z}, B_{z}^{\prime}$, of $z$ such that exactly one element of each block is a singleton in $x$.

(v) There is a block $B_{z}$ in $z$ such that exactly two elements of $B_{z}$ are singletons in $x$.

Let $k=2$. First consider the case in the statement of the lemma, i.e., case (v) above. Let $a, b$, be the two elements of $B_{z}$ which are singletons in $x$, and let $B_{x}$ consist of all elements of $B_{z}$ except $a$ and $b$. (Thus $B_{z}=B_{x} \cup\{a, b\}$.) Write $x_{1}$ for the subpartition of $x$ obtained by removing the block $B_{x}$ (i.e, $x=x_{1} / B_{x}$ ). Then $\delta(c)=-\left(x<x_{1} /\left(B_{x} \cup\{a\}\right)<z\right)-\left(x<x_{1} /\left(B_{x} \cup\{b\}\right)<z\right)-\left(x<x_{1} / B_{x} /\{a, b\}<\right.$ 
$z)$. The first two chains in this expression are in $[\hat{0}, w]_{\mathcal{B}}$; the third one has a barred label on the second edge.

We leave it to the reader to check that the chain $x<y<z$ is not related in cohomology to chains with barred labels in cases (i)-(iv) if $k=2$, and in all cases (i)-(v) if $k \geq 3$.

Lemma (2.6). Suppose $(x<y<z)$ is an unrefinable chain of length 3 in $\Pi_{\left(k, 1^{n-k}\right)}$, and let $\delta$ be the coboundary operator of the interval $[x, z]$ of $\Pi_{\left(k, 1^{n-k}\right)}$. Suppose that the label sequence of $(x<y<z)$ consists of a barred label followed by an unbarred label. Let $c$ denote the chain $(x<z)$. Then $\delta(c)+(x<y<z)$ is a linear combination of chains whose label sequence consists of an unbarred label followed by a barred label.

Proof. The hypothesis implies that $y$ is obtained from $x$ by merging two nonsingleton blocks $B_{1}(x)$ and $B_{2}(x)$ of $x$. There are three cases to consider.

(i) The partition $z$ is obtained from $y$ by creating a new 2-block $\{a, b\}$ out of two singletons of $y$; in this case clearly $\delta(c)+(x<y<z)=-\left(x<y^{\prime}<z\right)$ where $y^{\prime}$ is obtained from $x$ by creating the new 2-block $\{a, b\}$;

(ii) the partition $z$ is obtained from $y$ by merging a singleton $a(y)$ of $y$ with a non-singleton block $B(y)$ of $y$, and $B(y) \neq B_{1}(x) \cup B_{2}(x)$. Then $B(y)$ must occur as a block $B_{3}(x)$ of $x$, and hence $\delta(c)+(x<y<z)=-\left(x<y^{\prime \prime}<z\right)$ where $y^{\prime \prime}$ is obtained from $x$ by merging the singleton $a(y)$ with the block $B_{3}(x)$;

(iii) the partition $z$ is obtained from $y$ by merging a singleton $a(y)$ of $y$ with nonsingleton block $B(y)$ of $y$, but now $B(y)=B_{1}(x) \cup B_{2}(x)$. Here $\delta(c)+(x<$ $y<z)=-\left(x<y_{1}<z\right)-\left(x<y_{2}<z\right)$ where $y_{1}$ is obtained from $x$ by merging the singleton $a(y)$ with the block $B_{1}(x)$, and $y_{2}$ is obtained from $x$ by merging the singleton $a(y)$ with the block $B_{2}(x)$.

In the first two cases $z$ is obtained from $y^{\prime}$ (respectively $y^{\prime \prime}$ ) by merging the two non-singleton blocks $B_{i}(x), i=1,2$. In the third case $z$ is obtained from $y_{1}$ (respectively from $y_{2}$ ) by merging the non-singleton blocks $\{a(y)\} \cup B_{1}(x)$ with $B_{2}(x)$ (respectively $\{a(y)\} \cup B_{2}(x)$ with $B_{1}(x)$ ). The conclusion follows.

Lemma (2.7). Let $k \geq 3$ and let $w$ be a partition in $\Pi_{\left(k, 1^{n-k}\right)}$ with no singleton blocks. Then the cohomology relations in $\widetilde{H}^{*}(\hat{0}, w)_{\mathcal{B}}$ coincide with the top cohomology relations in $\widetilde{H}^{*}(\hat{0}, w)$.

Proof. Immediate from the fact that all cohomology relations involve unrefinable chains of the same length, and for $k \geq 3$, maximal length chains in $[\hat{0}, w]$ must be in $[\hat{0}, w]_{\mathcal{B}}$.

Now let $V_{t}$ denote the vector subspace spanned by all decreasing chains whose pivot has $t$ blocks. Recall that $P_{t}$ is the set of integer partitions of $n$ with $t$ parts, all greater than or equal to $k$.

Theorem (2.8). For $\mu \in P_{t}$, let $W_{\mu}$ denote the $N_{S_{n}}\left(S_{\mu}\right)$-module

$$
\widetilde{H}^{*}(\hat{0}, \mu)_{\mathcal{B}} \otimes \widetilde{H}^{*}(\mu, \hat{1}) .
$$

(i) For $k \geq 3$, the $S_{n}$-module, $\widetilde{H}^{n-3-t(k-2)}\left(\Pi_{\left(k, 1^{n-k}\right)}\right)$ is isomorphic to

$$
\bigoplus_{\mu \in P_{t}} \operatorname{Ind}_{N_{S_{n}}\left(S_{\mu}\right)}^{S_{n}} W_{\mu}
$$


(ii) Define $L_{t}=\bigoplus_{j \geq t} V_{j}$. Then the spaces $L_{t}$ are $S_{n}$-invariant for all $t \geq 1$, and if $k=2$, the basis of decreasing chains gives the following filtration of the top cohomology:

$$
\widetilde{H}^{*}\left(\Pi_{\left(k, 1^{n-k}\right)}\right)=L_{1} \supset L_{2} \supset \cdots \supset L_{\left\lfloor\frac{n}{k}\right\rfloor} .
$$

For $k=2$ the quotient module $L_{t} / L_{t+1}$ is $S_{n}$-isomorphic to

$$
\bigoplus_{\mu \in P_{t}} \operatorname{Ind}_{N_{S_{n}}\left(S_{\mu}\right)}^{S_{n}} W_{\mu} .
$$

Consequently for all $k \geq 2$, as an $S_{n}$-module $\widetilde{H}^{*}\left(\Pi_{\left(k, 1^{n-k}\right)}\right)$ is a direct sum

$$
\bigoplus_{t=1}^{\left\lfloor\frac{n}{k}\right\rfloor} \bigoplus_{\mu \in P_{t}} \operatorname{Ind}_{N_{S_{n}}\left(S_{\mu}\right)}^{S_{n}} W_{\mu} .
$$

Proof. For $w \in \Pi_{\left(k, 1^{n-k}\right)}$ with no singleton blocks, let $V_{w}$ be the vector subspace of $\widetilde{H}^{*}\left(\Pi_{\left(k, 1^{n-k}\right)}\right)$ which is spanned by all decreasing chains with pivot $w$. From the basis of decreasing chains one sees that the following vector space decompositions hold:

$$
\widetilde{H}^{*}\left(\Pi_{\left(k, 1^{n-k}\right)}\right)=\bigoplus_{t \geq 1} V_{t}
$$

and

$$
\begin{aligned}
V_{t} & =\bigoplus_{\mu \in P_{t}} \bigoplus_{g \in S_{n} / N_{S_{n}}\left(S_{\mu}\right)} V_{g \cdot w_{\mu}}, \\
V_{w} & =\widetilde{H}^{*}(\hat{0}, w)_{\mathcal{B}} \otimes \widetilde{H}^{*}(w, \hat{1}) .
\end{aligned}
$$

(Here $g \cdot w_{\mu}$ denotes the image of $w_{\mu}$ under $g$.)

Let $c$ be a decreasing chain in $V_{w}$. Clearly the image of the chain $c$ by an element $g \in S_{n}$ has pivot $g \cdot w$, and both $c$ and its image by $g$ have the property that all labels preceding the pivot are unbarred.

(i) When $k \geq 3$, the decreasing chains of length $n-1-t(k-2)$ are precisely the ones in $V_{t}$, and hence $V_{t}$ is the cohomology of $\Pi_{\left(k, 1^{n-k}\right)}$ in degree $n-3-t(k-2)$.

In order to rewrite (straighten) the chain $g \cdot c$ in terms of the basis of decreasing chains, we use the cohomology relations. Lemmas 2.1, 2.2, 2.3 and 2.7 imply that in order to straighten the chain $g \cdot c$, we can work independently above and below the pivot (indeed, one straightens below the pivot by straightening in $[\hat{0}, g \cdot w]_{\mathcal{B}}$, and above the pivot by straightening in $[g \cdot w, \hat{1}])$. Hence we conclude that $g \cdot\left(V_{w}\right)=V_{g \cdot w}$. In particular, it follows that the space $V_{w_{\mu}}$ is $N_{S_{n}}\left(S_{\mu}\right)$-invariant; in view of (2.2), its $N_{S_{n}}\left(S_{\mu}\right)$-module structure is given by $W_{\mu}$. Because of the decomposition (2.1) and the fact that the spaces $V_{w}$ are permuted by the action of $S_{n}$, it follows that $\widetilde{H}^{n-3-t(k-2)}\left(\Pi_{\left(k, 1^{n-k}\right)}\right)$ is the induced module as stated.

(ii) For $k \geq 3$, since $V_{t}=\widetilde{H}^{n-3-t(k-2)}\left(\Pi_{\left(k, 1^{n-k}\right)}\right), V_{t}$ (and hence $L_{t}$ ) is $S_{n^{-}}$ invariant.

Now let $k=2$. Note that the subspace $V_{t}$ is not $S_{n}$-invariant, except in the case $t=\left\lfloor\frac{n}{k}\right\rfloor$. To show that $L_{t}$ is $S_{n}$-invariant, it is enough to take $g$ to be an adjacent transposition $(i, i+1)$. In this case, if the chain $g \cdot c$, where $c \in V_{t}$, fails to be 
decreasing, the unbarred labels fail to be decreasing by at most one inversion of consecutive labels.

Lemmas 2.1, 2.2, 2.5 and 2.6 imply that to straighten the chain $g \cdot c$ in cohomology, one works first below the pivot with $t$ blocks. Indeed, one iteration of Lemma 2.5 gives $g \cdot c=\beta+c^{\prime}$, where $\beta$ is a sum of decreasing chains with pivot $g \cdot w$. The chain $c^{\prime}$ is either zero or a chain which is a result of the straightening of type (v) in the proof of Lemma 2.5, and hence could have a barred label followed by an unbarred label. If $c^{\prime}=0$ then $g \cdot c \in V_{t}$.

Now suppose $c^{\prime} \neq 0$. It is easy to see that the unbarred labels of $c^{\prime}$ form a decreasing subsequence. If $c^{\prime}$ does not have a barred label followed by an unbarred label then it is a chain with pivot less than $g \cdot w$ whose labels are decreasing below the pivot. On the other hand, if $c^{\prime}$ has a barred label followed by an unbarred label then Lemma 2.6 shows that by successively working up the chain from the first element that is obtained by merging two non-singleton blocks, the cohomology relations can be applied to give, eventually, a linear combination of chains with pivot less than $g \cdot w$, having only unbarred labels below the pivot; these labels are decreasing because they form the same sequence as the unbarred labels of $c^{\prime}$. In either case (i.e., $c^{\prime}$ has a barred label followed by an unbarred label or $c^{\prime}$ does not), $c^{\prime}$ is a linear combination of chains with pivots less than $g \cdot w$, having decreasing unbarred labels below the pivot. By Lemmas 2.1 and 2.2 the straightening is now completed by working on each of these chains above the pivots. Since the pivots are all less than $g \cdot w$, they have more than $t$ blocks. It follows that $c^{\prime} \in L_{t}$. Hence $g \cdot c \in L_{t}$ and the spaces $L_{t}$ are indeed $S_{n}$-invariant.

From the preceding considerations it also follows that the matrices of the action of $S_{n}$ with respect to the basis of decreasing chains, ordered in increasing length of pivot, is block upper triangular (the basis of decreasing chains is divided into blocks according to the number of blocks in the pivot), and a little thought shows that the block diagonal portion coincides exactly with the action on the spaces $W_{\mu}$. The statement about the quotients now follows.

Hence we obtain the decomposition of $\widetilde{H}^{*}\left(\Pi_{\left(k, 1^{n-k}\right)}\right)$ for all $k \geq 2$.

\section{The Frobenius Characteristic of the homology RePRESENTATION}

Our next goal is to compute the representation of $S_{n}$ on the space

$$
\bigoplus_{\mu \in P_{t}} \operatorname{Ind}_{N_{S_{n}}\left(S_{\mu}\right)}^{S_{n}} W_{\mu}
$$

For an arbitrary partition $\lambda \vdash n$ we denote by $s_{\lambda}$ the irreducible $S_{n}$-representation indexed by $\lambda$. In particular for $n \geq k \geq 2$, we denote by $s_{\left(k, 1^{n-k}\right)}$ the irreducible representation of $S_{n}$ indexed by the hook shape $\left(k, 1^{n-k}\right)$. We write $h_{n}$ for the trivial representation $s_{(n)}$ and $e_{n}$ for the sign-representation $s_{\left(1^{n}\right)}$. Let $G$ be a finite group. If $V$ is an $S_{m}$-module and $W$ is a $G$-module, we write $V[W]$ for the wreath product module canonically induced by the representations $\otimes^{m} W$ of $\chi^{m} G$ and $V$ of $S_{m}$.

We shall need a lemma on the $S_{n}$-action on the cohomology of a product of $n$ posets. This lemma will be used to compute the representation on the cohomology of $\Pi_{\left(k, 1^{n-k}\right)}$ and on the Whitney cohomology. The proof below gives a precise combinatorial description of the sign twists occurring in the Whitney cohomology of various subposets of the partition lattice. (See also [Su2].)

For a poset $P$, denote by $P^{n}$ the $n$-fold product of $P$ with itself. 
Lemma (3.1). Let $P$ be a poset of length $r$. Let $S_{n}$ act on $P^{n}$ by permuting the coordinates and let $G$ be a finite group of automorphisms of $P$. Then the wreath product $S_{n}[G]$ acts on $\widetilde{H}^{n r-2}\left(P^{n}\right)$ by $e_{n}\left[\widetilde{H}^{r-2}(P)\right]$ if $r$ is odd and $h_{n}\left[\widetilde{H}^{r-2}(P)\right]$ if $r$ is even.

Proof. For the sake of simplicity, we give the proof for the case $n=2$. The easy modifications needed to extend the proof to general $n$ are left to the reader.

Since $P \times P$ has length $2 r$, only maximum length chains in $P \times P$ contribute to its cohomology in degree $2 r-2$. First note that any maximum length chain in $P \times P$ can be represented by a shuffle of two maximum length chains $c_{1}, c_{2}$ of $P$. For example, if $c_{1}$ is the maximum length chain $\left(\hat{0}<x_{1}<x_{2}<\hat{1}\right)$ and $c_{2}$ is the maximum length chain $\left(\hat{0}<y_{1}<y_{2}<\hat{1}\right)$, let $\sigma$ be the string 121221 of 1's and 2's. Then the triple $\left(c_{1}, c_{2} ; \sigma\right)$ corresponds to the maximum length chain $c=((\hat{0}, \hat{0})<$ $\left.\left(x_{1}, \hat{0}\right)<\left(x_{1}, y_{1}\right)<\left(x_{2}, y_{1}\right)<\left(x_{2}, y_{2}\right)<\left(x_{2}, \hat{1}\right)<(\hat{1}, \hat{1})\right)$. More precisely, the string of 1's and 2's encodes the shuffle of chains in the following manner: a maximum length chain $c=\left((\hat{0}, \hat{0})=\left(x_{0}, y_{0}\right)<\left(x_{1}, y_{1}\right)<\cdots<\left(x_{2 r}, y_{2 r}\right)=(\hat{1}, \hat{1})\right)$ in the product has the property that $x_{i} \leq x_{i+1}$ and $y_{i} \leq y_{i+1}$ for all $i$, with equality in exactly one component; hence the $x_{i}$ 's form a maximum length chain $c_{1}$ from $\hat{0}$ to $\hat{1}$ and the $y_{j}$ 's form a maximum length chain $c_{2}$ from $\hat{0}$ to $\hat{1}$ in $P$. The chain $c$ can be recovered from the chains $c_{1}$ and $c_{2}$ if we specify a string of $r$ 1's and $r 2$ 's, where a 1 in position $i$ indicates that $x_{i-1}<x_{i}$, while a 2 in position $i$ indicates that $y_{i-1}<y_{i}$.

Let $\left(c_{1}, c_{2} ; \sigma\right)$ be a maximum length chain in $P \times P$, where each $c_{i}$ is a maximum length chain from $\hat{0}$ to $\hat{1}$ in $P$, and $\sigma$ is a string consisting of $r$ 1's and $r 2$ 's. Let $\rho$ denote the canonical string of $r$ 1's followed by $r$ 2's. (By definition of $\rho$, the maximum length chain $\left(c_{1}, c_{2} ; \rho\right)$ in $P \times P$ passes through $(\hat{1}, \hat{0})$.) We claim that in cohomology we have the relation

$$
\left(c_{1}, c_{2} ; \sigma\right)=\operatorname{sgn}(\sigma)\left(c_{1}, c_{2} ; \rho\right),
$$

where the sign of $\sigma, \operatorname{sgn}(\sigma)$, is $(-1)$ to the number of times a 1 follows a 2 in $\sigma$. Given two maximum length chains $x_{0}<x_{1}<\cdots<x_{r}$ and $y_{0}<y_{1}<\cdots<y_{r}$ in $P$, one has the following relation between cohomology classes for maximal length chains in the product poset:

$$
\begin{aligned}
\left(\cdots<\left(x_{i}, y_{j}\right)<\right. & \left.\left(x_{i}, y_{j+1}\right)<\left(x_{i+1}, y_{j+1}\right)<\cdots\right) \\
& =-\left(\cdots<\left(x_{i}, y_{j}\right)<\left(x_{i+1}, y_{j}\right)<\left(x_{i+1}, y_{j+1}\right)<\cdots\right),
\end{aligned}
$$

because the sum of the two chains is the result of applying the coboundary operator to a chain of length $2 r-1$ of the form $\ldots<\left(x_{i}, y_{j}\right)<\left(x_{i+1}, y_{j+1}\right)<\cdots$. Note that the 1-2 strings of length $2 r$ associated to the two chains are respectively $\cdots 21 \cdots$ and $\cdots 12 \cdots$. Successive iteration of this relation establishes (3.1).

The transposition $\tau$ in $S_{2}$ switches the coordinates of an element $(x, y)$ in $P \times P$, and therefore sends the chain $c=\left(c_{1}, c_{2} ; \rho\right)$ to the chain $\tau c=\left(c_{2}, c_{1} ; \bar{\rho}\right)$, where $\bar{\rho}$ is the string with $r$ 2's followed by $r 1$ 's. But the sign of $\bar{\rho}$ is $(-1)^{r^{2}}$ and hence we have, for maximum length chains $c_{1}$ and $c_{2}$ of $P$,

$$
\tau\left(c_{1}, c_{2} ; \rho\right)=(-1)^{r^{2}}\left(c_{2}, c_{1} ; \rho\right) .
$$

Another type of cohomology relation on maximum length chains in $P \times P$ is obtained by applying the coboundary operator to length $2 r-1$ chains of the forms

$$
\cdots<\left(x_{i}, y_{j}\right)<\left(x_{i+2}, y_{j}\right)<\cdots
$$


and

$$
\cdots<\left(x_{i}, y_{j}\right)<\left(x_{i}, y_{j+2}\right)<\cdots .
$$

This yields respective cohomology relations

$$
\left(c_{1}, c_{2} ; \sigma\right)=-\sum\left(c_{1}^{\prime}, c_{2} ; \sigma\right)
$$

and

$$
\left(c_{1}, c_{2} ; \sigma\right)=-\sum\left(c_{1}, c_{2}^{\prime} ; \sigma\right)
$$

where the first sum ranges over all $c_{1}^{\prime}$ appearing as a summand in the cohomology relation $c_{1}=-\sum c_{1}^{\prime}$ and the second sum ranges over all $c_{2}^{\prime}$ appearing as a summand in the cohomology relation $c_{2}=-\sum c_{2}^{\prime}$. Clearly all the cohomology relations in $\widetilde{H}^{2 r-2}(P \times P)$ are generated by those given in (3.1), (3.4), and (3.5).

Cohomology relation (3.1) shows that the chains contributing to cohomology may be taken to be of the form $\left(c_{1}, c_{2} ; \rho\right)$, where $c_{1}$ and $c_{2}$ are maximum length chains in $P$. Write $c_{1} \cdot c_{2}$ for the cohomology class of the chain $\left(c_{1}, c_{2} ; \rho\right)$. It follows from (3.4) and (3.5) that $c_{1} \cdot c_{2}$ depends only on the cohomology classes of $c_{1}$ and $c_{2}$. Since (3.1), (3.4) and (3.5) generate all the cohomology relations in $\widetilde{H}^{2 r-2}(P \times P)$, we can conclude that $\widetilde{H}^{2 r-2}(P \times P)$ is isomorphic (as a vector space) to $\widetilde{H}^{r-2}(P) \otimes$ $\widetilde{H}^{r-2}(P)$. Clearly this isomorphism commutes with the action of $G \times G$.

It follows from (3.3) that

$$
\tau\left(c_{1} \cdot c_{2}\right)=(-1)^{r^{2}} c_{2} \cdot c_{1} .
$$

Hence the transposition $\tau$ acts trivially on $\widetilde{H}^{r-2}(P)^{\otimes 2}$ if $r$ is even, and like the sign if $r$ is odd, as claimed.

The notion of wreath product module $V[W]$ can be extended to virtual modules. Here we need only consider a special case: First let $V_{1}$ and $V_{2}$ be $S_{m}$-modules and $W$ be an $S_{c}$-module. Then $\left(V_{1}-V_{2}\right)[W]$ is defined to be the (possibly virtual) $S_{m}\left[S_{c}\right]$-module $V_{1}[W]-V_{2}[W]$, Now if $V$ is any (possibly virtual) $S_{m}$-module and $W$ is an $S_{c}$-module then $V[-W]$ is defined by

$$
V[-W]=(-1)^{m}\left(e_{m} \otimes V\right)[W] .
$$

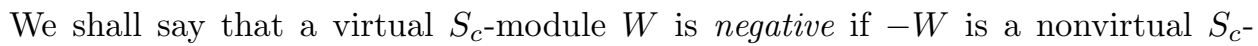
module.

For background on symmetric functions and plethysm, we refer to [Macd]. As in [Macd] we denote the Frobenius characteristic by ch. We shall need the following fact about wreath product modules: the characteristic $\operatorname{ch}\left(\operatorname{Ind}_{S_{m}\left[S_{c}\right]}^{S_{m c}}(V[W])\right)$ is the plethysm $\operatorname{ch}(V)[\operatorname{ch}(W)]$. Recall that the homogeneous symmetric function $h_{n}$ and the elementary symmetric function $e_{n}$ are the characteristics of the trivial and sign representations of $S_{n}$, respectively, while the Schur function $s_{\lambda}$ is the characteristic of the irreducible indexed by the integer partition $\lambda$. We will frequently use the same notation for both a (virtual) representation of $S_{n}$ and its Frobenius characteristic. In particular, we use the notation $h_{n}, e_{n}$ and $s_{\lambda}$ for both the corresponding irreducible representation and its Frobenius characteristic (i.e., the Schur function for this shape).

We need some notation and results of [Su1, Section 1], which we review here briefly. 
Let $\mu$ be an integer partition of $n$ with $t$ parts and let $m_{i}$ denote the multiplicity of the part $i$ in $\mu$. For any (possibly virtual) representation $\gamma$ of $S_{t}$, and any sequence of (possibly negative) representations $R_{i}$ of $S_{i}$, denote by

$$
\left(\operatorname{Res}_{\times_{j} S_{m_{j}}} \gamma\right)\left[\bigotimes_{i} R_{i}\right]
$$

the representation of the normaliser $N_{S_{n}}\left(S_{\mu}\right)$ defined as follows: Suppose the restriction

$$
\left(\operatorname{Res}_{\times_{j} S_{m_{j}}} \gamma\right)
$$

decomposes into irreducibles for $\left(S_{m_{1}} \times \cdots \times S_{m_{i}} \times \cdots\right)$ so that the irreducible indexed by the vector of partitions $\mu=\left(\mu^{1}, \ldots, \mu^{i}, \ldots\right)$ appears with multiplicity $c_{\underline{\mu}}$. Then

$$
\left(\operatorname{Res}_{\times_{j} S_{m_{j}}} \gamma\right)\left[\bigotimes_{i} R_{i}\right]
$$

denotes the (possibly virtual) representation

$$
\bigoplus_{\underline{\mu}} c_{\underline{\mu}} s_{\mu^{1}}\left[R_{1}\right] \otimes s_{\mu^{2}}\left[R_{2}\right] \otimes \cdots \otimes s_{\mu^{i}}\left[R_{i}\right] \otimes \cdots .
$$

We also need the following facts.

Lemma (3.2) [Su1, Lemma 1.5]. Let $Q$ be a (possibly virtual) representation of $S_{r}$ and let $f$ and $g$ be (possibly negative) representations of $S_{a}$ and $S_{b}$, respectively. Then the degree $n$ term in the plethysm $\operatorname{ch} Q[\operatorname{ch~} f+\operatorname{ch~} g]$ is the Frobenius characteristic of

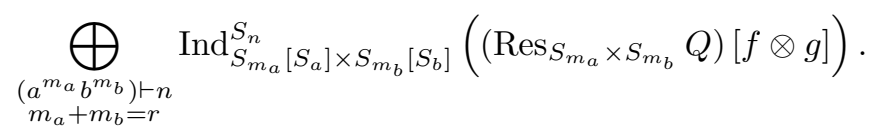

Lemma (3.3) [Su1, Remark 2.10.1]. Let $\mu$ be an integer partition of $n$ with $t$ parts, and let $m_{i}$ denote the multiplicity of the part $i$. Let $f$ and $g$ be (possibly virtual) representations of $S_{t}$ and let $\rho_{i}$ and $\tau_{i}$ be two series of (possibly negative) representations of $S_{i}, i \geq 1$. Then the inner tensor product of $N_{S_{n}}\left(S_{\mu}\right)$-modules corresponding to

$$
\left(\operatorname{Res}_{\times_{i} S_{m_{i}}} f\right)\left[\rho_{1} \otimes \rho_{2} \otimes \cdots\right]
$$

and

$$
\left(\operatorname{Res}_{\times_{i} S_{m_{i}}} g\right)\left[\tau_{1} \otimes \tau_{2} \otimes \cdots\right]
$$

evaluates according to the rule:

$$
\left(\operatorname{Res}_{\times_{i} S_{m_{i}}} f\right)\left[\bigotimes_{i} \rho_{i}\right] \otimes\left(\operatorname{Res}_{\times_{i} S_{m_{i}}} g\right)\left[\bigotimes_{i} \tau_{i}\right]=\left(\operatorname{Res}_{\times_{i} S_{m_{i}}} f \otimes g\right)\left[\left(\bigotimes_{i} \rho_{i} \otimes \tau_{i}\right)\right]
$$

We write $\pi_{n}$ for the characteristic of the $S_{n}$-representation afforded by the homology $\widetilde{H}_{*}\left(\Pi_{n}\right)$ of the full partition lattice $\Pi_{n}$. 
Proposition (3.4) [Su1, proof of Theorem 1.4]. Let $\mu$ be a partition of $n$ with $t$ parts, and let $m_{i}$ denote the multiplicity of the part $i$. Then the $N_{S_{n}}\left(S_{\mu}\right)$-module $\widetilde{H}_{*}(\mu, \hat{1})$ is given by

$$
\left(\operatorname{Res}_{\times_{j} S_{m_{j}}} \pi_{t}\right)\left[\bigotimes_{j} h_{j}\right]
$$

Now we are ready to present our main result.

Theorem (3.5). (i) Let $3 \leq k \leq n$. For $1 \leq t \leq\left\lfloor\frac{n}{k}\right\rfloor$, the Frobenius characteristic of the (co)homology of $\Pi_{\left(k, 1^{n-k}\right)}$ in degree $n-3-t(k-2)$ is given by the degree $n$ term in the plethysm

$$
(-1)^{n-t(k-1)} \pi_{t}\left[\sum_{j \geq k}(-1)^{j-k+1} s_{\left(k, 1^{j-k}\right)}\right] .
$$

(ii) Let $2=k \leq n$. Then all the (co)homology of $\Pi_{\left(k, 1^{n-k}\right)}=\Pi_{n}$ is concentrated in degree $(n-3)$ and its characteristic $\pi_{n}$ is given by the degree $n$ term in the sum of plethysms

$$
\sum_{t=1}^{\left\lfloor\frac{n}{2}\right\rfloor}(-1)^{n-t} \pi_{t}\left[\sum_{j \geq 2}(-1)^{j+1} s_{\left(2,1^{j-2}\right)}\right] .
$$

Proof. By Theorem 2.8, we need to determine the $N_{S_{n}}\left(S_{\mu}\right)$-module structure of the space $W_{\mu}$. First we consider the $N_{S_{n}}\left(S_{\mu}\right)$-module structure of the cohomology of the subposet $[\hat{0}, \mu]_{\mathcal{B}}$. By Lemma 2.3 (i) and Lemma 3.1, it is enough to know the $S_{r}$-module structure of the homology of the truncated Boolean subposet $B_{r}^{\geq k}$. This was computed by Solomon [So] to be the irreducible representation indexed by the hook $\left(k, 1^{r-k}\right)$. Lemma 3.1 applies to the subposet $[\hat{0}, \mu]_{\mathcal{B}}$, giving the structure of the $N_{S_{n}}\left(S_{\mu}\right)$-module $\widetilde{H}^{*}(\hat{0}, \mu)_{\mathcal{B}}$, to be

$$
\bigotimes_{\substack{j \geq k \\ j-k \text { odd }}} h_{m_{j}}\left[s_{\left(k, 1^{j-k}\right)}\right] \otimes \bigotimes_{\substack{j \geq k \\ j-k \text { even }}} e_{m_{j}}\left[s_{\left(k, 1^{j-k}\right)}\right]
$$

where $m_{j}$ is the multiplicity of the part $j$ of $\mu$. Using (3.4) we have

$$
h_{m_{j}}\left[(-1)^{j-k+1} \cdot s_{\left(k, 1^{j-k}\right)}\right]= \begin{cases}h_{m_{j}}\left[s_{\left(k, 1^{j-k}\right)}\right], & \text { if } j-k \text { is odd } ; \\ (-1)^{m_{j}} \cdot e_{m_{j}}\left[s_{\left(k, 1^{j-k}\right)}\right], & \text { otherwise. }\end{cases}
$$

Hence $\widetilde{H}^{*}(\hat{0}, \mu)_{\mathcal{B}}$ can be rewritten as

$$
(-1)^{n-t(k-1)} \cdot\left(\operatorname{Res}_{\times_{i} S_{m_{j}}} h_{t}\right)\left[\bigotimes_{j \geq k}(-1)^{j-k+1} s_{\left(k, 1^{j-k}\right)}\right],
$$

where $t$ is the number of parts of the integer partition $\mu$ of $n$. The exponent is explained by observing that, modulo 2 ,

$$
\sum_{j-k \text { even }} m_{j} \equiv \sum_{j \geq k} m_{j}(j-k+1)=\sum_{j \geq k} j m_{j}-(k-1) \sum_{j \geq k} m_{j} .
$$

Now use the facts that $\sum_{j} m_{j}=t$ and $\sum_{j} j m_{j}=n$. 
From Proposition 3.4 , the $N_{S_{n}}\left(S_{\mu}\right)$-module $\widetilde{H}^{*}(\mu, \hat{1})$ is given by

$$
\left(\operatorname{Res}_{\times_{j} S_{m_{j}}} \pi_{t}\right)\left[\bigotimes_{j \geq k} h_{j}\right]
$$

Hence, by Lemma 3.3 , the $N_{S_{n}}\left(S_{\mu}\right)$-module $W_{\mu}=\widetilde{H}^{*}(\hat{0}, \mu)_{\mathcal{B}} \otimes \widetilde{H}^{*}(\mu, \hat{1})$ is

$$
(-1)^{n-t(k-1)} \cdot\left(\operatorname{Res}_{\times_{i} S_{m_{j}}} \pi_{t}\right)\left[\bigotimes_{j \geq k}(-1)^{j-k+1} s_{\left(k, 1^{j-k}\right)}\right] .
$$

By Lemma 3.2, the characteristic of $\bigoplus_{\mu \in P_{t}} \operatorname{Ind}_{N_{S_{n}}\left(S_{\mu}\right)}^{S_{n}} W_{\mu}$ is thus the degree $n$ term in

$$
(-1)^{n-t(k-1)} \pi_{t}\left[\sum_{j \geq k}(-1)^{j-k+1} s_{\left(k, 1^{j-k}\right)}\right] .
$$

The statements of parts (i) and (ii) now follow from the respective parts of Theorem 2.8.

The virtual components of the preceding expression are conveniently eliminated by tensoring with the sign representation. As in [Macd], $\omega$ is the involution on the ring of symmetric functions which corresponds to this operation: $\omega\left(h_{n}\right)=e_{n}$.

Corollary (3.6). (i) Let $3 \leq k \leq n$. For $1 \leq t \leq\left\lfloor\frac{n}{k}\right\rfloor$, the characteristic of $\tilde{H}_{n-3-t(k-2)}\left(\Pi_{\left(k, 1^{n-k}\right)}\right)$ tensored with the sign representation is given by the degree $n$ term in the plethysm

$$
\left(\omega^{k+1} \pi_{t}\right)\left[\sum_{j \geq k} s_{\left(j-k+1,1^{k-1}\right)}\right]
$$

(ii) Let $2=k \leq n$. Then the characteristic of $\tilde{H}_{n-3}\left(\Pi_{n}\right)$ tensored with the sign representation is given by the degree $n$ term in the sum of plethysms

$$
\sum_{t=1}^{\left\lfloor\frac{n}{2}\right\rfloor}\left(\omega \pi_{t}\right)\left[\sum_{j \geq 2} s_{(j-1,1)}\right]
$$

Proof. By using the rules of plethysm,

$$
f[-g]=(-1)^{\operatorname{deg} f} \omega(f)[g]
$$

and

$$
\omega(f[g])=\left(\omega^{\operatorname{deg} g}(f)\right)[\omega(g)],
$$

where $f$ is homogeneous in the first equation and $g$ is homogeneous in the second equation (see [Macd]), one can establish the following general rule of plethysm: If $f$ is a homogeneous symmetric function of degree $h$ and $g_{i}$ is a homogeneous symmetric function of degree $i$ for $i=1,2, \ldots$, then for any $r \geq 0$

$$
\left.\omega\left(f\left[\sum_{i \geq 1}(-1)^{i+r} g_{i}\right]\right)\right|_{\operatorname{deg} n}=\left.(-1)^{n+h r} w^{r}(f)\left[\sum_{i \geq 1} w\left(g_{i}\right)\right]\right|_{\operatorname{deg} n} .
$$


By applying $\omega$ to the plethystic expressions in each of the parts of Theorem 3.5, and invoking this general rule of plethysm, we obtain the respective parts of the corollary.

The same techniques can be used to determine the $S_{n}$-module structure of the Whitney homology of $\Pi_{\left(k, 1^{n-k}\right)}$. For the purposes of this paper we shall use the following description of Whitney homology, due to Björner ([Bj2, Theorem 5.1]): the Whitney homology in degree $p>0$, of any poset $P$ with minimum element $\hat{0}$, is given by

$$
W H_{p}(P)=\bigoplus_{x \in P} \widetilde{H}_{p-2}(\hat{0}, x)
$$

Whitney cohomology $W H^{p}(P)$ is defined similarly, with cohomology replacing homology. Note that $W H_{p}\left(\Pi_{\left(k, 1^{n-k}\right)}\right)$ and $W H^{p}\left(\Pi_{\left(k, 1^{n-k}\right)}\right)$ are isomorphic $S_{n^{-}}$ modules.

Theorem (3.7). The contribution in degree $p>0$ of the representation on the Whitney (co)homology of $\Pi_{\left(k, 1^{n-k}\right)}$, tensored with the sign, is given by the degree $n$ term in

$$
\sum_{r, t \geq 1} \omega^{k}\left(\left.e_{r}\left[\sum_{i \geq 1} \omega\left(\pi_{i}\right)\right]\right|_{\operatorname{deg} t}\right)\left[\sum_{j \geq k} s_{\left(j-k+1,1^{k-1}\right)}\right] e_{n-p-r-t(k-2)},
$$

where the outer sum ranges over all $r$ and $t$ such that $r+t(k-2) \leq n-p$.

Proof. Consider a lower interval $[\hat{0}, x]$ in $\Pi_{\left(k, 1^{n-k}\right)}$. Let $x$ have $r$ blocks of size $\geq k$, and $s$ singletons. The decreasing chains $c$ in $[\hat{0}, x]$ are in one-to-one correspondence with pairs of decreasing chains $\left(c_{1}, c_{2}\right)$, as follows: Let $w_{c}$ be the first element in the chain $c$ which has the same set of singletons as $x$. Assume $w_{c}$ has $t$ non-singleton blocks. Note that the non-singleton blocks form a set partition of $n-s$ elements. Then $c_{1}$ is the portion of the chain $c$ in $\left[\hat{0}, w_{c}\right]$ and $c_{2}$ is the portion of the chain in $\left[w_{c}, \hat{1}\right]$. As in Lemma 2.1, $c_{1}$ is in fact a decreasing chain in $\left[\hat{0}, w_{c}\right]_{\mathcal{B}}$, which is again isomorphic to a product of truncated Boolean lattices. Also $\left[w_{c}, x\right]$ is isomorphic to a lower interval of the form $[\hat{0}, y]$, for some $y$ of rank $t-r$, in $\Pi_{t}$. Hence we have topological and representation-theoretic decompositions as in Proposition 2.4 and Theorem 2.8:

$$
W H^{p}\left(\Pi_{\left(k, 1^{n-k}\right)}\right)=\bigoplus_{w_{c}}\left(\widetilde{H}^{*}\left(\hat{0}, w_{c}\right)_{\mathcal{B}} \otimes \bigoplus_{x \geq w_{c}} \widetilde{H}^{*}\left(w_{c}, x\right)\right),
$$

where $w_{c}$ has $t$ non-singleton blocks, $x$ has $r \leq t$ non-singleton blocks, and both have the same set of $s$ singletons. Note that the second factor in the tensor product is isomorphic (as a vector space) to the Whitney cohomology of $\Pi_{t}$ in degree $t-r$. If the non-singleton blocks of $w_{c}$ have sizes $b_{1}, \ldots, b_{t}$ then $\left[\hat{0}, w_{c}\right]$ contributes chains of length $\sum_{i=1}^{t}\left(b_{i}-k+1\right)$, while $\left[w_{c}, x\right]$ contributes chains of length $t-r$. Hence the sum above runs over all $w_{c}$ and $x$ such that $p=\sum_{i=1}^{t}\left(b_{i}-k+1\right)+t-r=n-s-r-t(k-2)$.

Making this $S_{n}$-equivariant as in Theorem 2.8, we find that the representation on $W H^{p}\left(\Pi_{\left(k, 1^{n-k}\right)}\right)$ decomposes into

$$
\bigoplus_{\nu} \operatorname{Ind}_{N_{S_{n}}\left(S_{\nu}\right)}^{S_{n}}\left(B^{\nu} \otimes P^{\nu}\right)
$$


for some spaces $B^{\nu}$ and $P^{\nu}$; here the sum runs over all integer partitions $\nu$ of $n$ with $s$ parts equal to 1 and $t$ parts all greater than or equal to $k$, such that $s+t(k-2)<n-p$. In order to describe the modules $B^{\nu}$ and $P^{\nu}$, let $\mu$ be the partition of $n-s$ consisting of the parts of $\nu$ not equal to 1 . Then $B^{\nu}$ is the (outer) tensor product of the trivial $S_{s}$-module with $\widetilde{H}^{*}(\hat{0}, \mu)_{\mathcal{B}}$; similarly $P^{\nu}$ is the tensor product of the trivial $S_{s}$-module with representation of $N_{S_{n-s}}\left(S_{\mu}\right)$ obtained by restricting the action on the Whitney cohomology of $\Pi_{t}$ in degree $t-r$ to the Young subgroup $\chi_{i} S_{m_{i}}$, where $r=n-p-s-t(k-2)$ and $m_{i}$ is the multiplicity of the part $i$ in $\mu$. Using (3.7) in the proof of Theorem 3.5, we have

$$
B^{\nu}=(-1)^{n-s-t(k-1)} \cdot\left(\operatorname{Res}_{\times_{j} S_{m_{j}}} h_{t}\right)\left[\bigotimes_{j \geq k}(-1)^{j-k+1} s_{\left(k, 1^{j-k}\right)}\right] \otimes h_{s} .
$$

The equivariant structure of the Whitney cohomology of the partition lattice may be obtained by combining Lehrer and Solomon's determination of the equivariant structure of the cohomology of the complement of the braid arrangement [Le-So, Theorem 4.5] with [O-So, Corollary 5.7]. (Theorem 1.7 of [Su1] provides a direct proof of the $S_{n}$-module structure of the Whitney cohomology of $\Pi_{n}$. Lemma 3.1 provides an even easier proof.) The following formulation of this result, given in [Su1, Remark 1.8.1], is used in the present computation: The $S_{t}$-module structure of Whitney cohomology of $\Pi_{t}$ in degree $t-r$ is given by $\left.\omega\left(e_{r}\left[\sum_{i \geq 1} \omega\left(\pi_{i}\right)\right]\right)\right|_{\operatorname{deg} t}$. As in Proposition 3.4, we obtain

$$
P^{\nu}=\operatorname{Res}_{\times_{i} S_{m_{j}}} \omega\left(\left.e_{r}\left[\sum_{i \geq 1} \omega\left(\pi_{i}\right)\right]\right|_{\operatorname{deg} t}\right)\left[\bigotimes_{j \geq k} h_{j}\right] \otimes h_{s}
$$

Lemma 3.3 now yields

$$
\begin{aligned}
& B^{\nu} \otimes P^{\nu}=(-1)^{n-s-t(k-1)} \\
& \quad \cdot \operatorname{Res}_{\times_{j} S_{m_{j}}} \omega\left(\left.e_{r}\left[\sum_{i \geq 1} \omega\left(\pi_{i}\right)\right]\right|_{\operatorname{deg} t}\right)\left[\bigotimes_{j \geq k}(-1)^{j-k+1} s_{\left(k, 1^{j-k}\right)}\right] \otimes h_{s} .
\end{aligned}
$$

By (3.9) and Lemma 3.2, the Frobenius characteristic of $W H^{p}\left(\Pi_{\left(k, 1^{n-k}\right)}\right)$ can be expressed as the degree $n$ term in

$$
\sum_{r, t \geq 1}(-1)^{p+r+t} \omega\left(\left.e_{r}\left[\sum_{i \geq 1} \omega\left(\pi_{i}\right)\right]\right|_{\operatorname{deg} t}\right)\left[\sum_{j \geq k}(-1)^{j-k+1} s_{\left(k, 1^{j-k}\right)}\right] h_{n-p-r-t(k-2)}
$$

where the outer sum ranges over all $r$ and $t$ such that $r+t(k-2) \leq n-p$. By applying $\omega$ to the plethystic expression and using the general rule of plethysm (3.8), we obtain the result.

The above expression can be used to examine the occurrence of the trivial representation in the Whitney homology. This is applied in $[\mathrm{SW}]$ to the study of certain orbit spaces. 


\section{The $S_{n}$-MOdule $\widetilde{H}_{*}\left(\Pi_{\left(k, 1^{n-k}\right)}\right)$ AND THE DERIVED SERIES OF THE FREE LIE ALGEBRA}

In this section we collect some assorted facts about the cohomology of $\Pi_{\left(k, 1^{n-k}\right)}$ as an $S_{n}$-module. The most interesting of these is a remarkable connection, in the case $k=2$, between the filtration in cohomology provided by the basis of decreasing chains and the filtration of the free Lie algebra by derived series. The representation of $S_{n}$ on the multilinear component of this filtration was computed by Reutenauer ( $[\mathrm{Re}]$ ); we show that it coincides with the $S_{n}$-invariant filtration of the cohomology of $\Pi_{\left(k, 1^{n-k}\right)}$ arising from the spaces $L_{t}$ of Theorem 2.8 (ii). This filtration leads to a curious decomposition of the regular representation (Corollary 4.5).

We also show that for general $k$, the restriction of the $S_{n}$-module $\widetilde{H}_{*}\left(\Pi_{\left(k, 1^{n-k}\right)}\right)$ to $S_{n-1}$ may be expressed simply as a sum of outer tensor products of irreducibles of hook shape.

We begin by examining the case $k=2$. Here $\Pi_{\left(k, 1^{n-k}\right)}$ is the full partition lattice and all cohomology is in degree $(n-3)$. Corollary 3.6 is, in fact, the following result of Reutenauer in disguise:

Corollary (4.1) [Re]. Let $\ell_{n}$ denote the characteristic of the $S_{n}$-representation on the multilinear component of the free Lie algebra on $n$ generators. Then $\ell_{n}$ is given by the degree $n$ term in

$$
\sum_{t=1}^{\left\lfloor\frac{n}{2}\right\rfloor} \ell_{t}\left[\sum_{j \geq 2} s_{(j-1,1)}\right]
$$

Proof. It is well known that $\ell_{n}=\omega\left(\pi_{n}\right)$ (see eg. [Jo, Bar, Wa2]).

Reutenauer obtains his decomposition by considering the filtration of the free Lie algebra arising from its derived series, and computing a plethystic formula for the characteristic of each piece. In the cohomology of the partition lattice, this is the filtration of Theorem 2.8 (ii): the quotient $L_{t} / L_{t+1}$, when tensored with the sign, has characteristic given by the degree $n$ term in $\ell_{t}\left[\sum_{j \geq 2} s_{(j-1,1)}\right]$. In particular $L_{1} / L_{2}$ is the irreducible $s_{\left(2,1^{n-2}\right)}$. In a subsequent paper a basis for the homology of $\Pi_{\left(k, 1^{n-k}\right)}$ will be presented which, in the case $k=2$, gives a dual $S_{n}$-invariant filtration

$$
\tilde{H}_{*}\left(\Pi_{\left(2,1^{n-2}\right)}\right)=L_{1}^{\prime} \supset L_{2}^{\prime} \supset \cdots \supset L_{\left\lfloor\frac{n}{2}\right\rfloor}^{\prime},
$$

where the last term of the filtration, $L_{\left\lfloor\frac{n}{2}\right\rfloor}^{\prime}$, is the irreducible $s_{\left(2,1^{n-2}\right)}$.

We return now to the case of arbitrary $k \geq 2$.

For $1 \leq t \leq\left\lfloor\frac{n}{k}\right\rfloor$, denote by $H P_{n, k}^{t}$ the $S_{n}$-representation whose characteristic is given by the plethystic expression in Theorem 3.5 (i). If $k \geq 3$, this is the homology of $\Pi_{\left(k, 1^{n-k}\right)}$ in degree $n-3-t(k-2)$.

Proposition (4.2). The restriction of the $S_{n}$-module $H P_{n, k}^{t}$ to $S_{n-1}$ decomposes into a sum of induced modules as follows:

$$
\operatorname{ch}\left(\operatorname{Res}_{S_{n-1}}\left(H P_{n, k}^{t}\right)\right)=\sum_{\substack{\left(i_{1}, \ldots, i_{t}\right), i_{j} \geq k \\ i_{1}+\cdots+i_{t}=n}}\left(\prod_{r=1}^{t-1} s_{\left(k, 1^{i_{r}-k}\right)}\right) e_{i_{t}-k} h_{k-1} .
$$


Proof. By a standard fact about symmetric functions ([Macd]), $\operatorname{ch}\left(\operatorname{Res}_{S_{n-1}}\left(H P_{n, k}^{t}\right)\right)$ is obtained by taking the partial derivative with respect to the power-sum $p_{1}$ of $\operatorname{ch}\left(H P_{n, k}^{t}\right)$.

We also use the well-known result that the homology of $\Pi_{n}$ as an $S_{n-1}$-module is the regular representation ( [St, Theorem 7.6]). From Theorem 3.5 we find that the required characteristic is the degree $n$ term in

$$
(-1)^{n-t(k-1)}\left(\sum_{j \geq k}(-1)^{j-k+1} s_{\left(k, 1^{j-k}\right)}\right)^{t-1}\left(\sum_{j \geq k}(-1)^{j-k+1} e_{j-k} h_{k-1}\right) .
$$

Extracting the term of degree $n$, the result follows.

Corollary (4.3). For $k \geq 3$, the $S_{n-1}$-module $\tilde{H}_{n-3-t(k-2)}\left(\Pi_{\left(k, 1^{n-k}\right)}\right)$ has characteristic

$$
\sum_{\substack{\left(i_{1}, \ldots, i_{t}\right), i_{j} \geq k \\ i_{1}+\cdots+i_{t}=n}}\left(\prod_{r=1}^{t-1} s_{\left(k, 1^{i_{r}-k}\right)}\right) e_{i_{t}-k} h_{k-1}
$$

Remark (4.4). One can give a direct combinatorial proof of Corollary 4.3 by examining the action of $S_{n-1}$ on the basis of decreasing chains. This will be discussed in a future paper.

The next result also follows directly from Reutenauer's theorem.

Corollary (4.5). In the particular case $k=2$ we obtain the following decomposition of the regular representation of $S_{n}$ :

$$
p_{1}^{n}=\sum_{t=1}^{1+\left\lfloor\frac{n}{2}\right\rfloor} \sum_{\substack{i_{1}, \ldots, i_{t} \geq 2 \\ i_{1}+\cdots+i_{t}=n+2}}\left(\prod_{r=1}^{t-1} s_{\left(2,1^{i_{r}-2}\right)} e_{i_{t}-2}\right) .
$$

Note that the sign representation occurs in the summand corresponding to $t=$ 1, while the trivial representation occurs at the other extreme, in the summand corresponding to $t=1+\left\lfloor\frac{n}{2}\right\rfloor$.

We conclude with some easily deduced facts about character values and irreducibles occurring in $H P_{n, k}^{t}$.

Proposition (4.6). Let $n>k$. Let $\chi_{n, k}$ denote the character of $S_{n}$ acting on the whole homology $\widetilde{H}_{*}\left(\Pi_{\left(k, 1^{n-k}\right)}\right)$.

(i) The irreducibles appearing in $H P_{n, k}^{t}$ are indexed by partitions $\lambda$ of $n$ such that $k \leq \lambda_{1} \leq \min (t k, n-1)$. In particular the trivial and sign representations do not appear in the homology of $\Pi_{\left(k, 1^{n-k}\right)}$.

(ii) The irreducible indexed by $\left(k, 1^{n-k}\right)$ appears exactly once for $t=1$, and nowhere else.

(iii) The value of the character $\chi_{n, k}$ on a permutation of cycle-type $(1, \nu)$ where $\nu$ is any partition of $n-1$, is zero unless the partition $\nu$ is a refinement of the partition $(k-1, n-k)$.

(iv) The value of the character $\chi_{n, k}$ on an $n$-cycle is given by

$$
\sum_{d \mid n, d \leq \frac{n}{k}} \mu(d)(-1)^{n-k d} .
$$


Proof. Parts (i) and (ii) are consequences of the well-known fact (e.g., [St, Theorem 7.3]) that the representation $\pi_{t}$ is contained in the regular representation of $S_{t}$. Alternatively, they follow directly from Proposition 4.2.

Part (iii) is immediate from Proposition 4.2.

For part (iv), recall Hanlon's formula for the character values of $\pi_{t}$, (see [Ha1, Theorem 4]),

$$
\pi_{t}=\frac{1}{t} \sum_{d \mid t} \mu(d)(-1)^{t-\frac{t}{d}} p_{d}^{\frac{t}{d}}
$$

(As before $p_{d}$ denotes the $d$ th power-sum.) Now use the fact that the character value of the $S_{j}$-irreducible indexed by the hook $\left(k, 1^{j-k}\right)$ on a $j$-cycle is $(-1)^{j-k}$, and extract the terms involving $p_{n}$ in the plethysm of Theorem 3.5 (i).

\section{REFERENCES}

[Ar] Arnol'd, V.I.: The cohomology ring of the colored braid group, Math. Notes 5(1969), 138-140. MR 39:3529

[Bac] Baclawski, K.: Whitney numbers of geometric lattices, Advances in Math. 16(1975), 125-138. MR 52:7933

[Bar] Barcelo, H.: On the action of the symmetric group on the free Lie algebra and on the partition lattice, J. of Comb. Theory (A), 55(1990), 93-129. MR 91i:05118

[Bj1] Björner, A.: Shellable and Cohen-Macaulay partially ordered sets. Trans. Amer. Math. Soc. 260(1980), 159-183. MR 81i:06001

[Bj2] Björner, A.: On the homology of geometric lattices, Algebra Universalis 14(1982), 107-128. MR 83d:05029

[Bj-Lo-Y] Björner, A., Lovász, L., Yao, A.: Linear decision trees: volume estimates and topological bounds. In: Proc. 24th ACM Symp. on Theory of Computing, New York: ACM Press 1992, pp. 170-177.

[Bj-Wa] Björner, A., Wachs, M.L.: Shellable nonpure complexes and posets I, Trans. Amer. Math. Soc., 348(1996), 1299-1327. MR 96i:06008

[Bj-We] Björner, A., Welker, V.: Homology of the " $k$-equal" manifolds and related partition lattices, Advances in Math. 110 (No. 2) (1995), 277-313. MR 95m:52029

[Br] Brieskorn, E.: Sur les groupes de tresses. In: Séminaire Bourbaki 1971/72, Berlin, Heidelberg, New York: Springer 1973, pp. 21-44. MR 54:10660

[Ha1] Hanlon, P.: The fixed-point partition lattices, Pacific J. Math. 96(1981), 319-341. MR 83d:06010

[Ha2] Hanlon, P.: The Generalized Dowling lattices, Trans. AMS 325(1991), 1-37. MR 91h:06011

[Jo] Joyal, A.: Foncteurs analytiques et espèces de structures, in Lecture Notes in Mathematics 1234, Springer-Verlag, Berlin/Heidelberg/New York 1986, pp. 126-160. MR 89b:05014

[Le-So] Lehrer, G., Solomon, L.: On the action of the symmetric group on the cohomology of the complement of its reflecting hyperplanes, J. Algebra 104(1986), 410-424. MR 88a:32017

[Macd] Macdonald, I.: Symmetric functions and Hall polynomials, Oxford University Press 1979. MR 84g:05003

[O-So] Orlik, P., Solomon, L.: Combinatorics and topology of complements of hyperplanes, Inventiones Math. 56(1980), 167-189. MR 81e:32015

[Re] Reutenauer, C.: Dimensions and characters of the derived series of the free Lie-algebra. In: Lothaire, M. (ed.), Mots, Paris: Hermés 1990, pp. 171-182. MR 95c:17010

[So] Solomon, L.: A decomposition of the group algebra of a finite Coxeter group, J. Algebra 9(1968), 220-239. MR 38:1191

[St] Stanley, R. P.: Some aspects of groups acting on finite posets, Journal of Combinatorial Theory Ser. A 32(1982), 132-161. MR 83d:06002 
[Su1] Sundaram, S.: The homology representations of the symmetric group on CohenMacaulay subposets of the partition lattice, Advances in Math. 104 (No.2) (1994), 225-296. MR 96c:05189

[Su2] Sundaram, S.: Applications of the Hopf trace formula to computing homology representations, Proceedings of the Jerusalem Combinatorics Conference (May 1993), Contemporary Math. Series 178, Amer. Math. Soc. (1994), 277-309. MR 96f:05193

[SW] Sundaram, S. and Welker, V.: Groups actions on arrangements of linear subspaces and applications to configuration spaces, Trans. Amer. Math. Soc., to appear. CMP 95:15

[Wa1] Wachs, M. L.: A basis for the homology of the $d$-divisible partition lattice, Advances in Math.117(1996), 294-318. CMP 96:07

[Wa2] Wachs, M. L.: On the (co)homology of the partition lattice and the free Lie algebra, Proceedings of the 1994 Taormina Conference in honour of A. Garsia, Discr. Math., to appear.

Department of Mathematics, Wesleyan University, Middletown, Connecticut 06459

E-mail address: sheila@claude.math.wesleyan.edu

Department of Mathematics and Computer Science, University of Miami, Coral Gables, Florida 33124

E-mail address: wachs@math.miami.edu 